\title{
MODERN AND CONTEMPORARY CULTURAL HERITAGE DOCUMENTATION AND KNOWLEDGE BY SURVEYING AND ITS REPRESENTATION
}

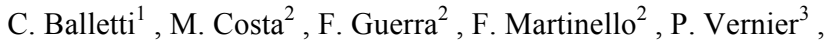 \\ Università IUAV di Venezia, 1 DPPAC, 2 DACC, 3 Sdl- Laboratorio di Fotogrammetria - (balletti, mcosta, guerra2, fmartinello, \\ vernier)@iuav.it; \\ Commission II, WG II/8
}

KEY WORDS: documentation, modern heritage, laser scanning, photogrammetry, 3d modelling, 3d print

\begin{abstract}
:
Conservation of modern and contemporary cultural heritage, which goes from design objects, to architecture, to cities and territories, is certainly a current topic and in the development phase as it is underway - in the same modernity - a process of systematic replacement of architectural elements, outcome of solutions then experimental, which today are reproduced with contemporary materials, analogous in the appearance, but intimately different especially in the technological content.

The paper describes the particular case of La Tour de Meudon, better known as The Tower, (1966) by André Bloc, a contemporary architect of Le Corbusier, founder of L'Architecture d'aujourd'hui, who created his habitable sculptures. All his works mark the evolution of geometric abstraction to the free form, and they are still admirable testimonies of a journey that led him from architecture to architecture. His Architecture and his sculpture intertwine, opening the plastic unity of form in physical space-time. The survey is a fundamental moment for the knowledge of these hybrid architectures, where the structural component is hidden by its evident plasticity, as if it were a large sculpture with abstract and overlapping geometric shapes.

Survey isn't only an analysis of geometries: it is instrumental to the other structural and material analyses since it provides a metric and topological basis on which to spatially locate the phenomena being studied. The integrated survey of the building (laser scanning, photogrammetry, topography) has allowed to document his project, contributing to the to definition of the actual construction characteristics and ascertain both the material consistency and the state of conservation.
\end{abstract}

\section{INTRODUCTION}

The Tower (formally called Sculpture-Habitacle 3, La Tour) by André Bloc (De Witt d.J. and De Witt E.R.,1987) located near Paris within the property of Meudon where it coexists with other works of the architect.

The brick and concrete Tower offers an open path similar to a vertical labyrinth, done by ramps and stairs. Although erected in 1966, the project started in 1964, the date of the first preparatory model. Two preparatory models in plaster allow to reconstruct the path followed by the genesis of the project. The first one (1964), corresponding to the earliest version, shows very small dimensions and a gentle treatment of volumes. It exhibits Bloc's will in disarticulating forms according to an upward movement to obtain a greater plastic expression, the fulcrum of the master's intentions.

These formal characteristics bring the architect closer to the maquettes dating back to 1963-64, called 'géométrie indiscipliné', which present aerodynamic shapes and reflect on the disjointed chaining of the volumes, in this case on a horizontal plane. The last model, extremely similar to the final realization, is dated 1965-66, realized in 1: 5 scale, it clearly distinguishes itself from the first one for its stable and massive appearance: the volumes acquire consistency, the surfaces become rough, the asymmetries reduced. The central core consists of a quadrangular structure, a stairwell of about $3.50 \mathrm{~m}$ that develops in open air on about 5 levels. To reach the top the proposed routes are multiple and not always reliable: corridors, stairs, blind alleys are entangled in a labyrinthine network, the absence of parapets and the presence of sloping ground parts make us completely lose the balance. The materials used are the same that we find in Sculpture-habitacle II: bricks with sporadic reinforcements in concrete; in this case the color is left to natural, the interior shines with different red depending on the sun's rays that penetrate through the surrounding vegetation.

The problem of the relationship between architecture and sculpture remains, but it is placed here in other terms: Bloc did not try to give a sculptural definition to architecture but the inverse. This sculpture is equivalent to an architecture, in terms of dimensions and constructive method, but it is suddenly transformed from 'object to be seen' to 'space to be traveled', urging to move from a contemplative status to an active one. From its 25 meters high and from its strategic position, the tower dominates the whole Bloc's property and admires the city of Paris.

The purpose of the survey was to obtain an accurate documentation suitable for a next phase of restoration and monitoring of the structure, in addition to a polygonal model to be printed (3D printing technology); the processed data and the model will be exposed in a exhibition about André Bloc and his works in Venice, organized by the researching group HeModern of the Iuav University, to which the authors belong, on April 2018 and later in Paris.

Considering that the Tower was the last work of Andrè Bloc, it was never surveyed and documented and, until today, only its maquettes was able to offer an idea of the constructive progress of this work. Thanks to the survey, carried out with well-established methods and techniques, and its representation it is possible to better understand the play of volumes, the fulls and the empties, the characteristics of this architecture such an organic form.

\section{SURVEYING LA TOUR OF MEUDON}

The integrated survey (topography, laser scanning, photogrammetry) allows to document the building's history, to define the current building features and to determinate the physical consistency and the state of conservation.

In planning restoration works for 20th century architecture, the implementation of digital survey is not so common, as most of the time the original project's documentation are still accessible and sufficient to inform restoration interventions. But it is clear that design drawings and the real architecture 
could never correspond because the project design represents a projection into the future, prefiguring time.

Surveying, as analysis, studies the form of the architecture considering the geometries of the building, and geometries have an essential role in the processes of conception, construction and comprehension which have involved the building over its lifetime (Balletti et al. 2017).

Surveying becomes a very complex operation: it requires critical interpretation abilities for a correct comprehension of the surveyed architecture from which the correct strategies can be derived to obtain the best result from the survey operations. As is well known (Balletti c. et al, 2014, Balletti c. et al, 2015, Remondino et al., 2010), many techniques are today available to generate $3 \mathrm{D}$ survey data, which are necessary to understand differences between design and reality. Digital photogrammetry and terrestrial laser scanning could be used to provide a greater number of measurements for similar object sizes, and therefore, are suitable to be integrated for more complex objects acquisition.

The adopted methodologies are well consolidated in the field of cultural heritage and they perform the better results for the knowledge of this peculiar architecture.

\subsection{Topographic survey}

According to the usual data acquisition workflow, all the data acquired by laser scanning and photogrammetry have to be georeferenced in the same local reference system (datum), which was defined through a topographic network, measured with a topographic Total Station Leica TCR 1103.

For the materialization of the main network it was not possible to create a closed polygonal because the site had many natural obstacles; for this reason it was decided to create a very simple geometric scheme of the topographical network: an isostatic triangle and an open polygonal point (Fig. 1). The cornerstones of the network were materialized in a non-permanent way through wooden stakes, engraved on the head with a marker for the correct centering of the instrument.

The following step consisted in surveying 37 targets placed on the external surface of the tower and on some surrounding natural points, to allow the referencing of laserscanning and photogrammetric survey data obtained in the same reference system. The observations were adjusted using Microsurvey StarNet software that enabled us to perform a least square adjustment, obtaining a standard deviation of $\pm 1 \mathrm{~mm}$ on the coordinates of the points.

\subsection{Laserscanning Survey}

At the same time, a part of the surveying team worked with the phase shift measurement Faro Focus 3D (www.faro.com) laser scanner (Fig. 2).

Acquisition phase was performed into 2 projects, one for the outer surfaces of the Tower and one for the inner part. Regarding the exteriors 10 scans were acquired with an angular pitch of $0.035^{\circ}$, so as to guarantee the acquisition of a point every $6 \mathrm{~mm}$ at a $10 \mathrm{~m}$ distance, with a density suitable for the 1:50 scale. The alligment and the registration of scans in topographical reference system was carried out within the Scene software, by automatic targets recognition. The acquisition of the inner part was more complicated, as the structure develops in elevation with a central stairwell with a labyrinthine outer path. Since the recording of the clouds of such a complex space was done on architectural points, not on targets, scans were acquired with a high overlap. It was therefore set an angular step of $0.070^{\circ}$ so as to acquire a point every $12 \mathrm{~mm}$ at $10 \mathrm{~m}$ of distance that when we would have superimposed all the scans we would have obtained a density of points adequate to scale 1:50.
For the inner part, 55 scans were acquired, obtaining a point cloud model consisting of 1100000000 points (Fig. 3).

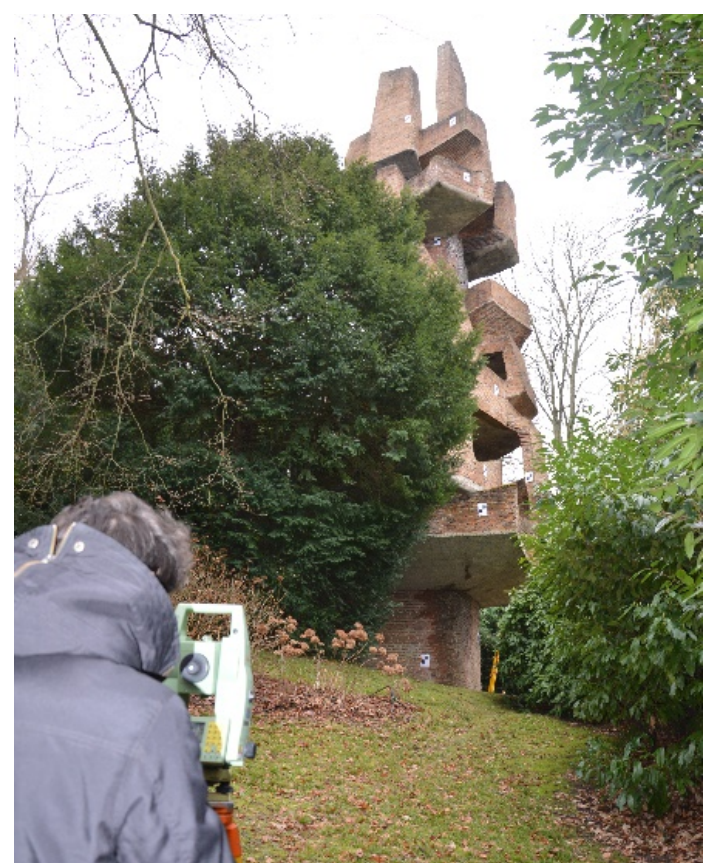

Figure 1 The topographical survey

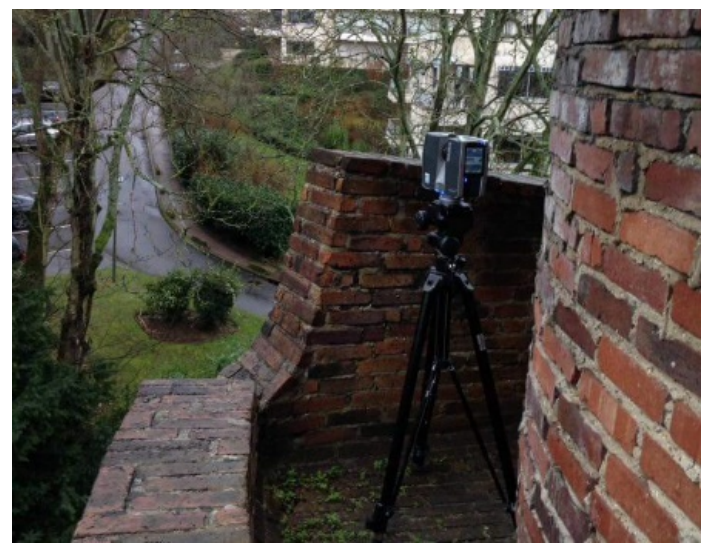

Figure 2 The Faro Focus 3D CAM2 acquiring scans of exteriors

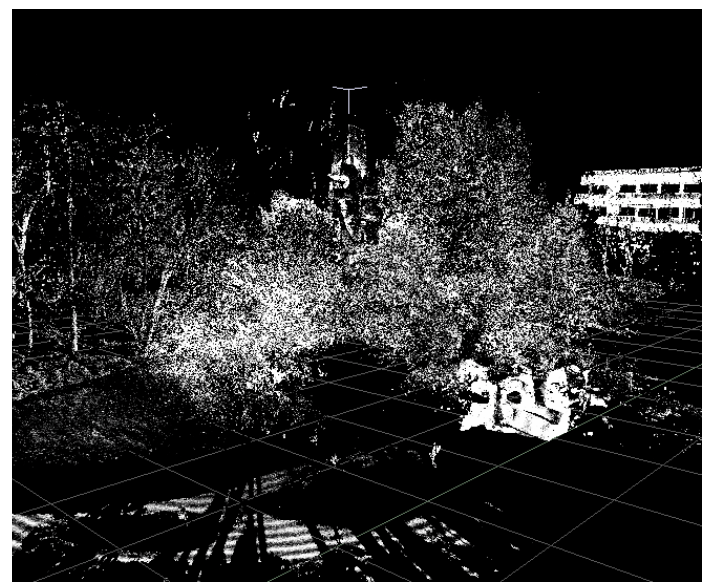

Figure 3 The points cloud final model 


\subsection{Photogrammetric survey}

The acquisition phase for the photogrammetric survey did not take place with the canonical schemes due to the vegetation that hindered regular setting patterns.

It was decided to proceed with the acquisition by using two SLR cameras for the exteriors, the Nikon D3200 and D610. For the inner surfaces a Sony rx100 was adopted, a compact camera mounted on a pole in order to acquire floors and missing exteriors, being able to lean outwards, and complete the model as much as possible.

Due to time constraints, it was decided to use a GoPro and a spherical camera in order to have an overabundance of data and be able to complete parts that could have presented a lack of data.

The first processed photogrammetric blocks were those of the two SLRs. Within the Agisoft Photoscan software we have created a chunck for each camera, so as to have two data to be able to subsequently compare and merge for the next phase which will concern the processing of data for a model suitable for 3D printing.

For the Nikon D3200 block, we have used 218 images, obtaining the texturized photogrammetric model (Fig. 4). The referencing took place after the texturing phase, importing the coordinates files and recognizing the markers on each frame (about 23 GCPs, Rsme minor than $0.0076 \mathrm{~m}$ ).

The second chunck, concerning the Nikon D610, included 50 frames and generated a model oriented obtaining a Rsme of $\pm 0.0058 \mathrm{~m}$, suitable for 1.50 scale. The model was registred by 18 GCPs.

As mentioned above, concerning the internal central compartment and the floorings, we used frames acquired by GoPro and Sony rx100. These two blocks were also processed separately so that the sw computation time was reduced. The Sony rx 100 project was based on 100 processed images allowing to build a mesh with a Rsme $\pm 0,0043 \mathrm{~m}$. In this case, being only the upper part, 6 GCPs were used. Instead the GoPro camera block was much more hardworking. The computation times were very high as it consists in processing 801 frames. Nevertheless, at the end of the process a Rsme of $\pm 0,0063 \mathrm{~m}$ was obtained, based on 23 GCPs.

In summary the projects/bocks obtained were:

- Nikon D3200: 218 frames; Rsme: \pm 0,0076 m, 23 GCPs;

- Nikon D610: 50 frames; Rsme : $\pm 0,0058 \mathrm{~m}$, 18 GCPs;

- Sony rx100: 100 frames; Rsme : $\pm 0,0043 \mathrm{~m}$, $6 \mathrm{GCP}$;

- GoPro: 801 frames; Rsme : $\pm 0,0063 \mathrm{~m}$, 23 GCPs

The photogrammetric data were used to integrate and fill the gaps of the laserscanning clouds, to obtain a model much more suitable for $3 \mathrm{D}$ printing.

\section{MAQUETTE}

Within the same place, just few meters from the tower, there was one $(3.5 \mathrm{~m}$ height) of the many maquettes (Fig. 5) that Bloc completed before the Tower was built. It was considered necessary for a better understanding of the work to survey both the Tower and its prototype, the maquette, in order compare the design idea and its realization. The creation of a polygonal surfaces model was done photogrammetrically, by using Agisoft Photoscan software, aligning 245 frames acquired with the Sony rx100 camera.
Once the alignment was processed it was possible to obtain a dense cloud consisting of 4771701 points, and create a triangulated mesh of 954340 faces. A laserscanning survey was also performed, thanks to which it was possible to fix the reference system necessary to orienting and scaling the photogrammetric model. 6 scans were acquired, aligned in Scene, by using 16 markers, and using the first scan as reference. Points cloud offered the coordinates of GCP employed in Photoscan performing the rototranslation with scale variation (Rsme: $\pm 0.0088 \mathrm{~m}$ ) (Fig. 6).

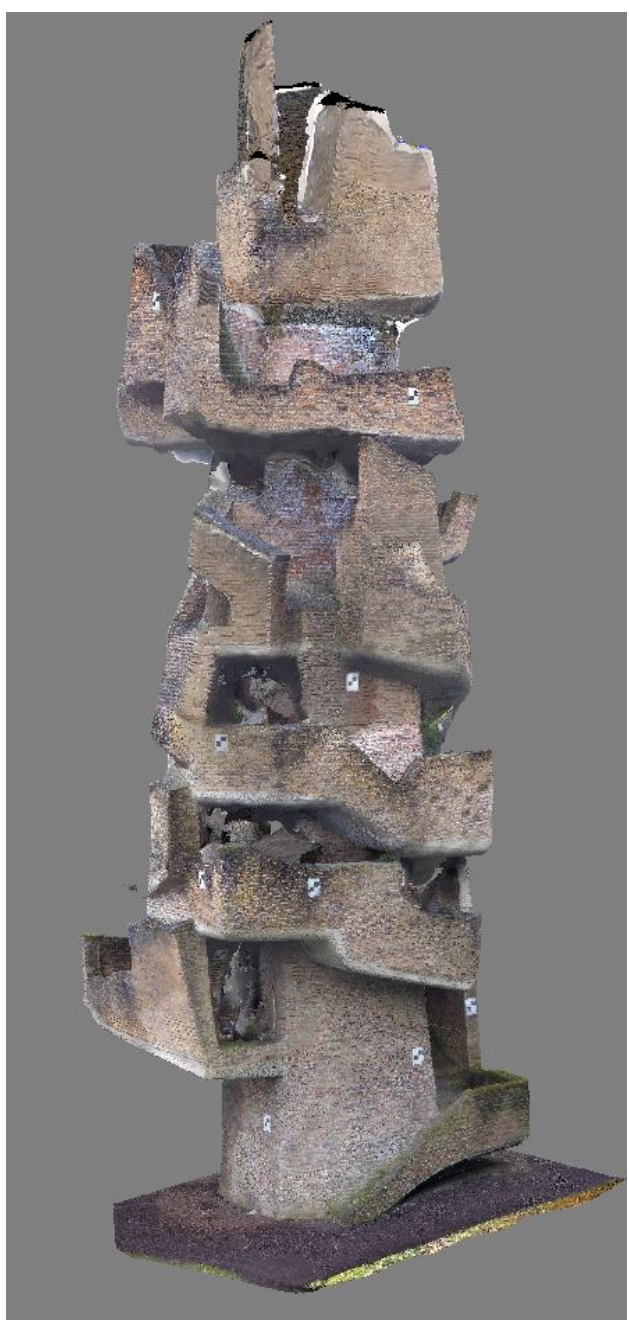

Figura 4 Texturized photogrammetric model

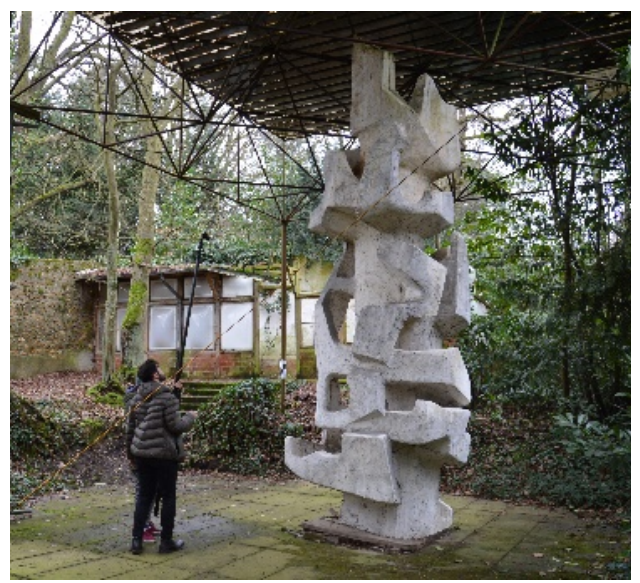

Figure 5 The Photogrammetric survey of the maquettes 


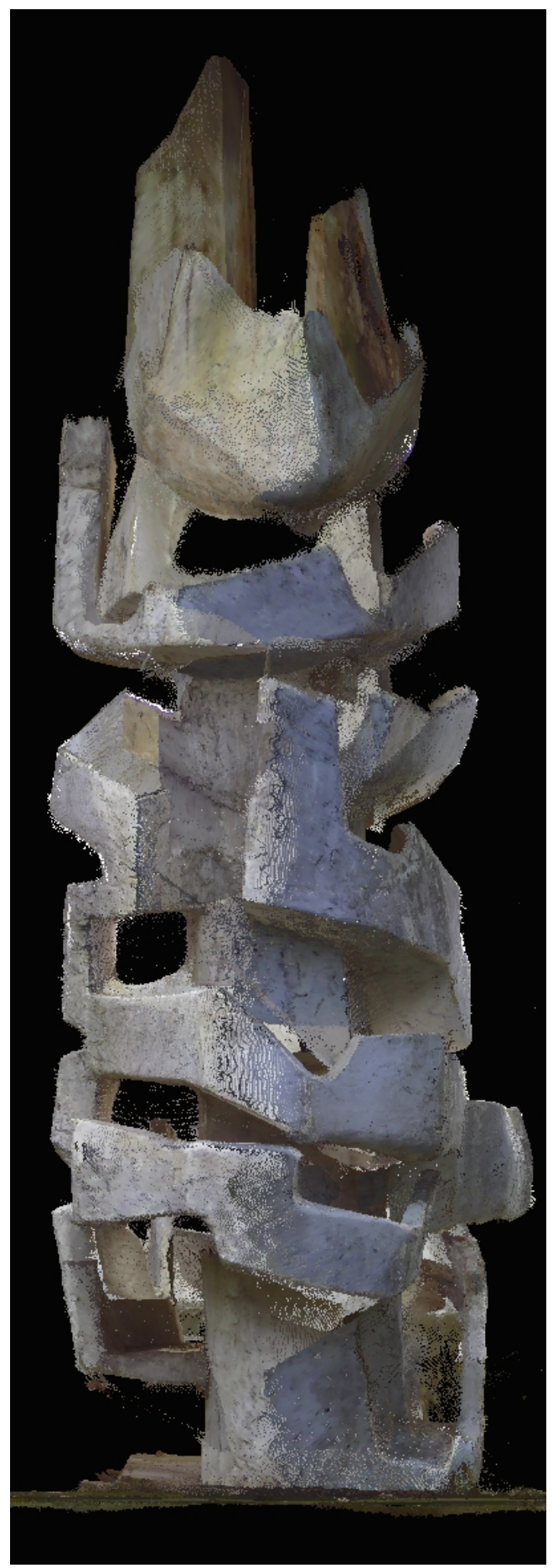

Figura 6 the Photogrammetric point cloud of the brick maquette

\section{3D PRINTING}

In the last years the development of 3D technologies applied to the field of Cultural Heritage has led to results of the utmost importance from the point of view of preservation, valorisation, communication and fruition of our assets. Between 3d technologies, solid printing has gained a special role in this development (Ballarin et al., 2017; Adami et al., 2015; Balletti et al., 2016; D'Agnano et al. 2015; Scopigno et al., 2015). The rapid creation of prototypes is a technique that allows the production of material copies of objects with complex geometries directly from the digital model in relatively short periods of time and often without being expensive (Neumüller et al.,2014).

One of the final purposes of this survey was to obtain a suitable and accurate digital model for a 3D printing of both the maquette and the Tower. For this reason the data obtained from the photogrammetric survey and laserscanning was further processed in Geomagic, a rapid prototyping software for $3 \mathrm{~d}$ models. In this environment, it was possible to combine, clean and complete parts where data was missing.

As for the maquette, the photogrametric mesh obtained in Photoscan was exported in STL ormat and imported into Geomagic and processed for the printing (Fig. 7).

The final model of the Tower was much more complicated.

After several tests, the solution was to process the data in two distinct parts, as two macro projects: one concerning the external part and another one concerning the interior.

The internal scans, oriented in a single reference system, were imported into Geomagic where they were cleaned and transformed into meshes individually.

Before merging the meshes, a forced alignment was carried out through the ICP algorithm and only after that it was possible to create a unique polygonal surface model of the entire central block.

Since the photogrammetric and laserscanning data was acquired with the same precision it was possible to integrate them.

In fact, the macro external project was created only by the Photoscan model through the usual cleaning and editing process in Geomagic.

Once the two macro projects were obtained, it was possible to create a single model, in STL format, with a precision of 1 $\mathrm{cm}$ and therefore suitable for the 1:50 scale ready for solid printing.

The model will be divided into several parts (Fig. 8) and printed by using a WASP printer, using a FFF technology, based on the stratification of liquid plastic materials. Casting is based on additive principle, so that each strata once deposited is covered by the following one, forming superimpositions which can create roughness, depending on the material, on the casting dimension, on the nozzle and on the casting precision.

\section{CONCLUSION}

Through the topographic survey, laserscanning, photogrammetric, metric and radiometric data were obtained, able to carry out surveys and studies for a restoration project. The Bloc's Tower was no documented before: no plants, no sections or elevations, in fact it was the first time that someone has detected its geometry.

Through the points cloud, photogrammetric or laser scanning, it was possible to extract profiles and to draw in CAD environment with a accurancy of $\pm 1 \mathrm{~cm}$ and therefore suitable for a 1:50 scale.

The software used for redrawing were Pointools or Recap to manage point clouds, Autocad for vector redraw.

About the $3 \mathrm{~d}$ printing, we are going to prints two phisical models: the first one of the Tower, in 1:20 scale, and the second one of maquette, whose size was calculated by making a fitting of the maquette respect to the tower model.

With this method we obtained two prints, one of the architecture and the other of the maquette, both of the same dimensions so as to be able to immediately compare the geometries and verify the differences between them.

The prints were made of white plastic material in such a way that it is possible to project, with the tecniques of video mapping, the textures of the original materials for a maximally mimetic scale representation. In addition to these 
projections, it is also planned to project colored textures at a later date to identify the correspondences and differences between the realized tower and the project maquette.

The two printed models will be an essential part of the exhibition which also includes the traditional vectorial drawings (plan, elevations and sections) particularly interesting for the Bloc's Tower which allow to capture both the tectonic and the figurative aspects of the opera.

It is important to consider that, if vertical sections are fundamental for the understanding of the work, this type of representation is completely extraneous to the project/design concept of Bloc's work, which, as already said, proceeded with the creation of the maquette sequences.

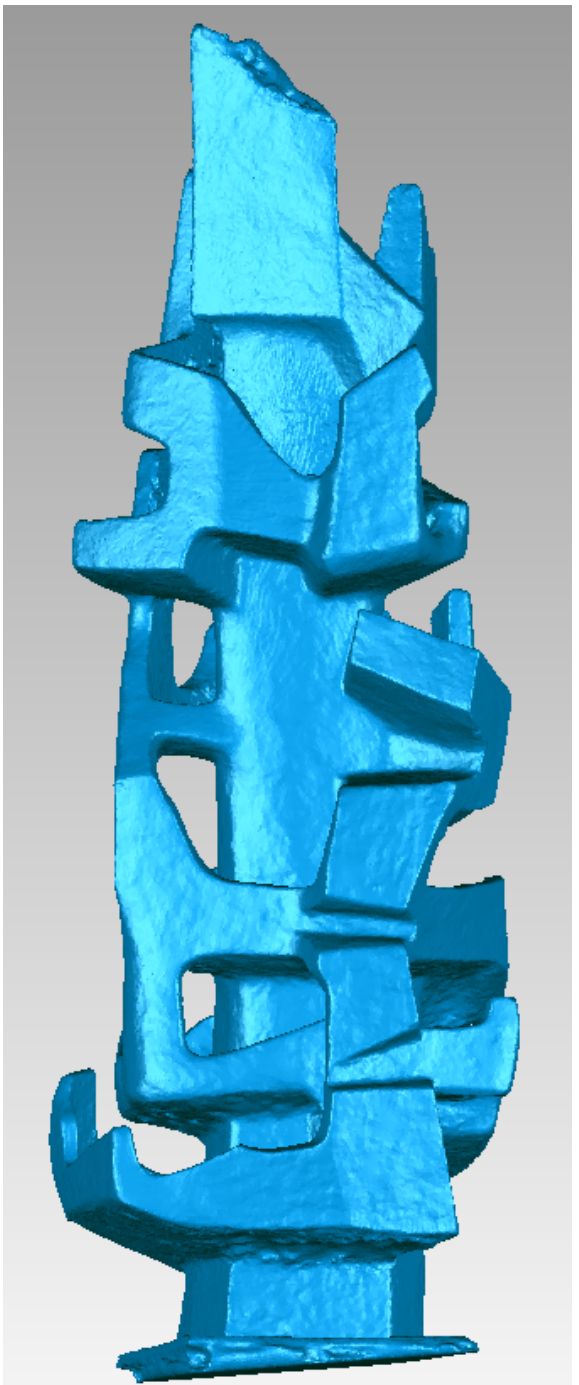

Figure 7 Final project STL

\section{REFERENCES}

Adami A., Balletti C., Fassi F., Fregonese L., Guerra F., Taffurelli L., Vernier P., 2015. The bust of Francesco II Gonzaga: from digital documentation to 3D printing. ISPRS Ann. Photogramm. Remote Sens. Spatial Inf. Sci., II-5/W3, pp. 915, doi:10.5194/isprsannals-II-5-W3-9-2015.

Balletti, C., Brussa, N., Gottardi, C., Guerra, F.: The documentation and reintegration of a lost past, ISPRS Ann. Photogramm. Remote Sens. Spatial Inf. Sci., II-5, 49-55, doi:10.5194/isprsannals-II-5-49-2014, 2014
Balletti C., D'Agnano F., Guerra F., Vernier P., 2016. From point cloud to digital fabrication: a tangible reconstruction of $\mathrm{Ca}$ ' Venier dei Leoni, the Guggenheim Museum in Venice. ISPRS Ann. Photogramm. Remote Sens. Spatial Inf. Sci., III5, pp. 43-49, doi:10.5194/isprs-annals-III-5-43-2016.

Balletti C., Guerra F., 2015. The survey of cultural heritage: a long story. Rendiconti Lincei, Vol. 26, pp. 115-125. doi: 10.1007/s12210-015-0411-8.

D’Agnano F., Balletti C., Guerra F., Vernier P., 2015. TOOTEKO: a case study of augmented reality for an accessibile cultural heritage. Digitization, 3D printing and sensors for audio-tactile experience. Int. Arch. Photogramm. Remote Sens. Spatial Inf. Sci., XL-5/W4, pp. 207-213, doi:10.5194/isprsarchives-XL-5-W4-2072015.

De Witt d.J. and De Witt E.R.,1987. Modern Architecture in Europe. Weidenfeld and Nicolson, London.

Neumüller M., Reichinger A., Rist F., Kern C., 2014. 3D Printing for Cultural Heritage: Preservation, Accessibility, Research and Education. Lecture notes in Computer Science. Springer, 8355, pp. 119-134. doi: 10.1007/978-3-6624463009.

Remondino F., Rizzi A., 2010. Reality-based 3d documentation of natural and cultural heritage sites techniques, problems and examples. Applied Geomatics, Vol.2(3), pp. 85-100.

Scopigno R., Cignoni P., Pietroni N., Callieri M., Dellepiane M., 2015. Digital Fabrication Techniques for Cultural Heritage: A Survey. Computer Graphics Forum, doi:10.1111/cgf.12781

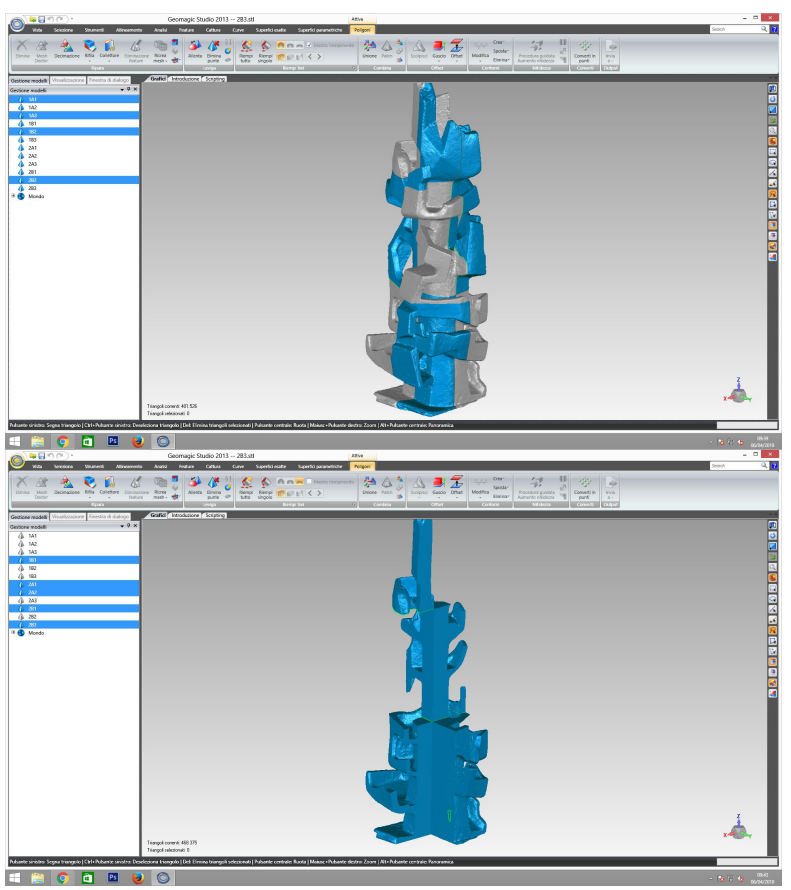

Figure 8. some of the parts in which the model to be printed has been divided 